\title{
Reconstruction of past Mediterranean climate: Unexplored sources of high resolution data in historic time
}

\author{
1st MedCLIVAR Workshop, Carmona, Spain, 8-11 November 2006
}

\author{
R. García-Herrera ${ }^{1}$, J. Luterbacher ${ }^{2}$, P. Lionello ${ }^{3}$, F. González-Rouco ${ }^{1}$, P. Ribera ${ }^{4}$, X. Rodó ${ }^{5}$, C. Kull ${ }^{6}$ and C. Zerefos ${ }^{7}$ \\ 'Facultad de CC Físicas, Universidad Complutense, Ciudad Universitaria, Madrid, Spain; rgarciah@fis.ucm.es \\ ${ }^{2}$ NCCR Climate, University of Bern, Switzerland \\ ${ }^{3}$ Department of Material Sciences, University of Lecce, Italy \\ ${ }^{4}$ Universidad Pablo de Olavide, Sevilla, Spain \\ ${ }^{5}$ Climate Research laboratory LRCPCB-UB, Barcelona, Catalunya (Spain) \\ ${ }^{6}$ PAGES IPO, Sulgeneckstrasse 38, 3007 Bern, Switzerland \\ University of Athens, Panepistimiopolis, Zografou, 157 84, Greece
}

This workshop was the first in a series of five meetings planned by MedCLIVAR (www.medclivar.eu), which is a program funded by the European Science Foundation (ESF) aiming to coordinate and promote research on different aspects of Mediterranean climate. The main MedCLIVAR activities include: (1) reconstruction of past climate, (2) description of patterns and mechanisms characterizing space-time variability, (3) comparisons of coupled climate model simulations with empirical reconstructions, (4) seasonal forecasting, and (5) identification of the forcing responsible for the observed changes.

The workshop was attended by 64 researchers from 15 countries (Fig. 1), 15\% being $\mathrm{PhD}$ students. The main purpose of the meeting was to identify sources of early instrumental data, and natural and documentary climate proxies, which had not been previously explored but which are relevant for the reconstruction of climate in the Mediterranean region during past millennia. The first session reviewed the availability of documentary sources in the larger Mediterranean region, with reports from different countries and archives. The second part of the workshop analyzed the availability of natural archives in the area, such as tree-rings, lake sediments, speleothems and mollusk shells, and their suitability to reconstructions of different climate parameters such as temperature, precipitation or drought. Several examples of modeling past Mediterranean climate were shown in the third session, with emphasis on the comparison between model outputs and proxy evidence.

The main conclusions can be summarized as follows:

The Mediterranean region is rich in natural and documentary proxies of past regional climate. They resolve different temporal and spatial scales and

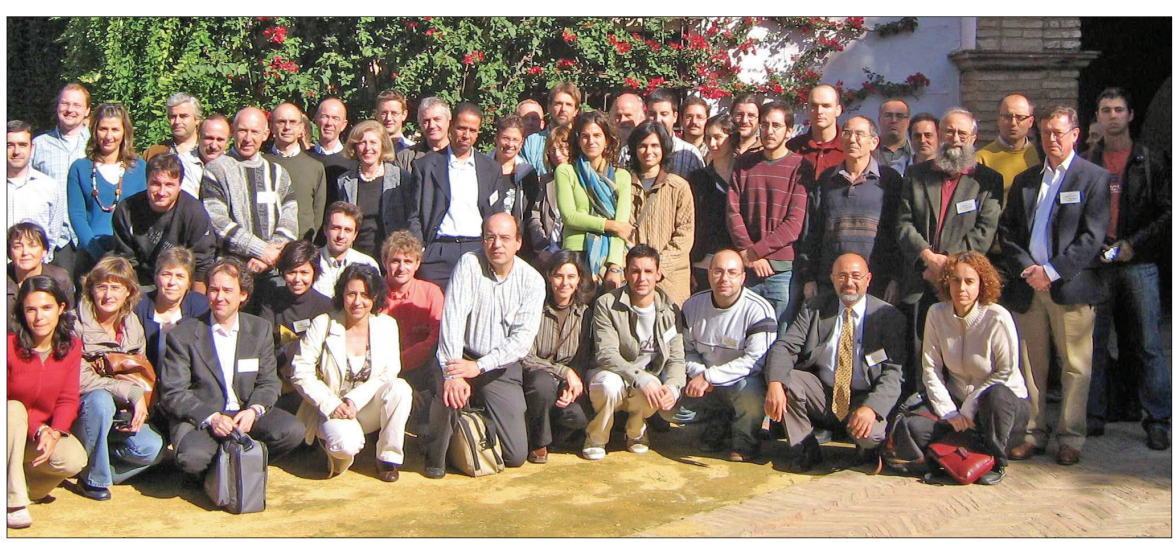

Figure 1: Participants at the $1^{\text {st }}$ MedCLIVAR workshop.

reflect a wide range of climate parameters (SST, salinity, temperature, precipitation, sea level, geochemistry, etc). However, the currently available proxy data sets are not sufficiently dense to reconstruct climate with a high spatial variability as would be required for the Mediterranean basin. The main gaps in information come from Northern Afri$\mathrm{ca}$, the Eastern Mediterranean and the Balkans.

There are numerous ongoing initiatives from different research teams to retrieve information from documentary collections and natural proxies but there is not a general strategy to search data and proxies on a basin-wide scale. This should be the target of an interdisciplinary approach, with researchers from different fields collaborating towards a common understanding of past climate change of the last 1000 to 2000 years using high-resolution proxies.

The simulation of past climates is accomplished using models of different complexity: EMICs and full complexity GCMs. Comparison with proxies at the Mediterranean scale is based on the use of GCMs. Simulations of the last millennium climate are subject to limitations by model resolution, orography representation, parameterization, as well as to model and external forcing uncertainties but still can provide some interesting comparisons with climate reconstructions.
Exercises comparing documentary and natural proxies, and model outputs are scarce for the region. They should improve the quality of all types of data sets and models but should be compared with care, taking into account the uncertainties in the proxy reconstructions, the complexity in the transfer functions from proxy parameters to climate variables, and the internal variability of the models, which is highly dependent on the modeled variable.

The workshop was the starting point towards building a community working together on the past climate of the Mediterranean region, incorporating scientists and data from the north African and Eastern Mediterranean communities, and creating links between paleoscientists, climatologists, and modelers. The next step will be the creation of a web-supported meta-database to increase data interchange. The database will be hosted by MedCLIVAR and will be supported by PAGES.

\section{Acknowledgements}

The workshop was generously supported by the ESF, PAGES, The Spanish Ministry of Science and Education, the National Observatory of Athens, and the University Pablo de Olavide of Spain. 